\title{
Periodicities of a System of Difference Equations
}

\author{
Weizhen Quan, Miaoqiao Pan, and Xiaopei Li \\ Basic Education School, Lingnan Normal University, Zhanjiang, Guangdong 524037, China \\ Correspondence should be addressed to Xiaopei Li; lixp27333@sina.com
}

Received 2 May 2017; Revised 3 August 2017; Accepted 1 October 2017; Published 24 December 2017

Academic Editor: Yong H. Wu

Copyright (C) 2017 Weizhen Quan et al. This is an open access article distributed under the Creative Commons Attribution License, which permits unrestricted use, distribution, and reproduction in any medium, provided the original work is properly cited.

We study the periodicities of a system of difference equations $x_{n+1}=\max \left\{1 / x_{n}, A_{n} / y_{n-k}\right\}, y_{n+1}=\max \left\{1 / y_{n}, B_{n} / x_{n-k}\right\}$, where initial values $\left(x_{-k}, y_{-k}\right), \ldots,\left(x_{0}, y_{0}\right) \in(0,+\infty) \times(0,+\infty)$. We show that if $A_{n}, B_{n} \in(0,1)$ are two periodic sequences, then every solution of the above system is eventually periodic with period 2. If $k$ is even, there must be one in $\left\{x_{n}\right\}$ and $\left\{y_{n}\right\}$ converges to period two solution.

\section{Introduction}

Difference equations are powerful tool that describe the law of nature. Recently, the theory of difference equations has received extensive attention because it can be applied to many areas of science and technology, such as the fields of information and e-science. Moreover, people pay more attention to the dynamics of max-type difference equations; they are concerned about the research of the period character of solutions and the convergence of positive solutions. Please refer to [1-7].

Szalkai [8] studied the max-type difference equation

$$
x_{n}=\max \left\{\frac{A_{0}}{x_{n}}, \frac{A_{1}}{x_{n-1}}, \ldots, \frac{A_{k}}{x_{n-k}}\right\}, \quad n=0,1,2, \ldots
$$

where $A_{i}(i=1,2, \ldots, k) \in \mathbb{R}$, and at least one of them is nonzero. The initial values $x_{0}, x_{-1}, \ldots, x_{-k}$ are nonzero real numbers, so every solution of this equation is periodic solution. Chen [9] studied the dynamics of max-type difference equation

$$
x_{n+1}=\max \left\{\frac{1}{x_{n}}, \frac{A_{n}}{x_{n-1}}\right\}, \quad n=0,1,2, \ldots,
$$

where $\left\{A_{n}\right\}_{n=0}^{\infty}=\{\ldots, \alpha, \beta, \alpha, \beta, \ldots\}$ is a two-cycle sequence, $\beta>\alpha>1$. The initial values $x_{0}, x_{-1}$ are nonzero real numbers; then every positive solution of this equation is bounded and persists, and $x_{n} \in[\sqrt{\alpha} / \beta, \beta \sqrt{\alpha}]$ for all $n \geq 0$.
Furthermore, every solution of this equation is periodic with period 4. Yang [10] studied the max-type difference equation

$$
x_{n}=\max \left\{\frac{1}{x_{n-1}^{\alpha}}, \frac{A}{x_{n-2}}\right\}, \quad n=0,1,2, \ldots,
$$

where $0<\alpha<1, A>0$; then every solution of this equation tends to be $\bar{x}=1$ or is eventually periodic with period 4 . Sun et al. [11] studied the dynamics of max-type difference equation

$$
x_{n+1}=\max \left\{\frac{1}{x_{n}}, \frac{A_{n}}{x_{n-1}}\right\}, \quad n=0,1,2, \ldots,
$$

where $\left\{A_{n}\right\}_{n=0}^{\infty}$ is a $k$-cycle sequence with $k \geq 2$. The initial values $x_{0}, x_{-1}$ are positive real numbers. Let $\lim _{\sup } \operatorname{si\infty }_{n \rightarrow \infty} x_{n}=$ $S$; if there exist infinitely many $n$ such that $x_{n} \geq S$ and $x_{n+1} \geq$ $S$, then $\left\{x_{n}\right\}$ is eventually equal to 1 . every positive solution of this equation is eventually periodic with period 2 provided $A_{n} \in(0,1)$.

Motivated by the work of above max-type difference equations, the aim of this paper is to investigate the periodicities of a system of max-type difference equations

$$
\begin{aligned}
& x_{n+1}=\max \left\{\frac{1}{x_{n}}, \frac{A_{n}}{y_{n-k}}\right\}, \\
& y_{n+1}=\max \left\{\frac{1}{y_{n}}, \frac{B_{n}}{x_{n-k}}\right\},
\end{aligned}
$$


where initial values $\left(x_{-k}, y_{-k}\right),\left(x_{-k+1}, y_{-k+1}\right), \ldots,\left(x_{0}, y_{0}\right) \in$ $(0,+\infty) \times(0,+\infty), k \in \mathbb{N}^{+}$, and $A_{n}, B_{n}$ are positive sequences. More precisely, we will prove that if $A_{n}, B_{n} \in$ $(0,1)$ are two periodic sequences, then every solution of $(5)$ is eventually periodic with period 2 . if $k$ is even, then one of $\left\{x_{n}\right\}$ and $\left\{y_{n}\right\}$ converges to a period two solution.

\section{Main Results}

Before proceeding with the proof of our main results in this section, we shall need the following lemmas.

Lemma 1. Let $\left\{\left(x_{n}, y_{n}\right)\right\}_{n=-k}^{+\infty}$ be a solution of system (5); then $x_{n+1} x_{n} \geq 1, y_{n+1} y_{n} \geq 1$, and $x_{n+1}+x_{n} \geq 2, y_{n+1}+y_{n} \geq 2$ for all $n \geq 0$.

Proof. By system (5), we have

$$
\begin{aligned}
& x_{n+1} \geq \frac{1}{x_{n}}, \\
& y_{n+1} \geq \frac{1}{y_{n}}
\end{aligned}
$$

for all $n \geq 0$. Hence $x_{n+1} x_{n} \geq 1, y_{n+1} y_{n} \geq 1$, and $x_{n+1}+x_{n} \geq$ $2 \sqrt{x_{n+1} x_{n}} \geq 2, y_{n+1}+y_{n} \geq 2 \sqrt{y_{n+1} y_{n}} \geq 2$. This completes the proof of the lemma.

Lemma 2. Let $\left\{\left(x_{n}, y_{n}\right)\right\}_{n=-k}^{+\infty}$ be a solution of system (5). If $n>$ $k$, then

$$
\begin{aligned}
& x_{n+1} \leq \max \left\{x_{n-1}, A_{n} y_{n-k-1}\right\} \leq \max \left\{x_{n-1}, y_{n-k-1}\right\}, \\
& y_{n+1} \leq \max \left\{y_{n-1}, B_{n} x_{n-k-1}\right\} \leq \max \left\{y_{n-1}, x_{n-k-1}\right\} .
\end{aligned}
$$

Proof. By Lemma 1 and $A_{n}<1$, we obtain that, for any $n>k$,

$$
\begin{aligned}
x_{n+1} & =\max \left\{\frac{x_{n-1}}{x_{n} x_{n-1}}, \frac{A_{n} y_{n-k-1}}{y_{n-k} y_{n-k-1}}\right\} \\
& \leq \max \left\{x_{n-1}, A_{n} y_{n-k-1}\right\} \leq \max \left\{x_{n-1}, y_{n-k-1}\right\} .
\end{aligned}
$$

Similarly, we also obtain that, for any $n>k$,

$$
\begin{aligned}
y_{n+1} & =\max \left\{\frac{y_{n-1}}{y_{n} y_{n-1}}, \frac{B_{n} x_{n-k-1}}{x_{n-k} x_{n-k-1}}\right\} \\
& \leq \max \left\{y_{n-1}, B_{n} x_{n-k-1}\right\} \leq \max \left\{y_{n-1}, x_{n-k-1}\right\} .
\end{aligned}
$$

This completes the proof of the lemma.

Theorem 3. Let $\left\{\left(x_{n}, y_{n}\right)\right\}_{n=-k}^{+\infty}$ be a solution of system (5) and $r_{n+1}=\max \left\{x_{n}, x_{n-1}, \ldots, x_{n-k-1}, y_{n}, y_{n-1}, \ldots, y_{n-k-1}\right\}$. Then

(1) $\left\{r_{n}\right\}$ is eventually monotonically decreasing. Moreover, if $T=\lim _{n \rightarrow \infty} r_{n}$, then $T \geq 1$;

(2) $\max \left\{\lim \sup _{n \rightarrow \infty} x_{n}, \lim \sup _{n \rightarrow \infty} y_{n}\right\}=T$.

Proof. (1) By Lemma 2, if $n>k$, we have

$$
\begin{aligned}
x_{n+1} & \leq \max \left\{x_{n-1}, y_{n-k-1}\right\} \\
& \leq \max \left\{x_{n}, x_{n-1}, \ldots, x_{n-k-1}, y_{n}, y_{n-1}, \ldots, y_{n-k-1}\right\} \\
& =r_{n+1} .
\end{aligned}
$$

Similarly, we obtain $y_{n+1} \leq r_{n+1}$. Hence

$$
\begin{aligned}
r_{n+2} & =\max \left\{x_{n+1}, x_{n}, \ldots, x_{n-k}, y_{n+1}, y_{n}, \ldots, y_{n-k}\right\} \\
& \leq r_{n+1},
\end{aligned}
$$

so $\left\{r_{n}\right\}$ is eventually monotonically decreasing. On the other hand, by Lemma 1 , we know $x_{n+1} x_{n} \geq 1, y_{n+1} y_{n} \geq 1$, so $r_{n} \geq 1$. Hence $T \geq 1$.

(2) It is easy to know that there are infinitely many $n$ such that $x_{n}=r_{n+1}$ or $y_{n}=r_{n+1}$, so $\lim \sup _{n \rightarrow \infty} x_{n} \geq T$, or $\lim \sup _{n \rightarrow \infty} y_{n} \geq T$. On the other hand, $x_{n} \leq r_{n+1}$ and $y_{n} \leq r_{n+1}$. Hence $\lim \sup _{n \rightarrow \infty} x_{n} \leq T$ and $\lim \sup _{n \rightarrow \infty} y_{n} \leq T$. Thus

$$
\max \left\{\limsup _{n \rightarrow \infty} x_{n}, \limsup _{n \rightarrow \infty} y_{n}\right\}=T .
$$

This completes the proof of the theorem.

Lemma 4. Let $a=\max \left\{A_{n}, B_{n}: n \in \mathbb{N}^{+}\right\}$and $\eta=((1-$ a)/2)T. Then there exists $N \in \mathbb{N}^{+}$such that

$$
\begin{aligned}
& x_{n+1} \leq \max \left\{x_{n-1}, T-\eta\right\}, \\
& y_{n+1} \leq \max \left\{y_{n-1}, T-\eta\right\},
\end{aligned}
$$

for all $n \geq N$.

Proof. Let $\eta_{1}=((1-a) / 2 a) T$. By Theorem 3, there exists $N \in$ $\mathbb{N}^{+}$such that $n-k-1 \geq N$ :

$$
y_{n-k-1}<T+\eta_{1}=\frac{1+a}{2 a} T .
$$

Then $A_{n} y_{n-k-1}<a((1+a) / 2 a) T=T-((1-a) / 2) T=T-\eta$. By Lemma 2, it follows that

$$
x_{n+1} \leq \max \left\{x_{n-1}, T-\eta\right\} .
$$

Similarly, we obtain $y_{n+1} \leq \max \left\{y_{n-1}, T-\eta\right\}$. This completes the proof of the lemma.

Lemma 5. There exists a positive integer $N_{1} \in \mathbb{N}^{+}$such that, for all $t \geq 0, x_{N_{1}+2 t} \geq x_{N_{1}+2 t+2} \geq T$ (if $\limsup _{n \rightarrow \infty} x_{n}=T$ ), or $y_{N_{1}+2 t} \geq y_{N_{1}+2 t+2} \geq T$ (if $\lim \sup _{n \rightarrow \infty} y_{n}=T$ ).

Proof. Let $N$ and $\eta$ be as Lemma 4. By Theorem 3, if $\lim \sup _{n \rightarrow \infty} x_{n}=T$, then there exists a monotonically increasing sequence $n_{t} \rightarrow+\infty$ such that $x_{n_{t}} \geq T$. On the other hand, there exists a subsequence $\left\{n_{t_{j}}\right\}$ of $\left\{n_{t}\right\}$ such that $n_{t_{j}}=$ $2 l_{t_{j}}+r(r \in\{0,1\})$. Without loss of generality, we may assume that $\exists N \in \mathbb{N}^{+}, n_{t}=2 l_{t}+r \geq N$ (for all $n_{t}$ ). By Lemma 4 , we get

$$
\begin{aligned}
T \leq x_{n_{t}} & =x_{2 l_{t}+r} \leq \max \left\{x_{2\left(l_{t}-1\right)+r}, T-\eta\right\} \\
& =x_{2\left(l_{t}-1\right)+r} \leq \max \left\{x_{2\left(l_{t}-2\right)+r}, T-\eta\right\} \\
& =x_{2\left(l_{t}-2\right)+r} \leq \max \left\{x_{2\left(l_{t}-3\right)+r}, T-\eta\right\} \\
& =\cdots=x_{2 l_{t-1}+r} .
\end{aligned}
$$

Write $N_{1}=2 l_{1}+r$. Then $x_{N_{1}+2 t} \geq x_{N_{1}+2 t+2} \geq T$ for all $t \geq 0$.

Similarly, we obtain that if $\lim _{\sup _{n \rightarrow \infty}} y_{n}=T$, then $y_{N_{1}+2 t} \geq y_{N_{1}+2 t+2} \geq T$. This completes the proof of the Lemma. 
Lemma 6. (1) If $\lim \sup _{n \rightarrow \infty} x_{n}=T$, then there exists an integer $N \in \mathbb{N}^{+}$such that both the number sequences $\left\{x_{N+2 t}\right\}$ and $\left\{y_{N+2 t+k+1}\right\}$ are monotonically decreasing, and $x_{N+2 t} x_{N+2 t-1}=$ 1, $y_{N+2 t+k} y_{N+2 t+k+1}=1$ for all $t \geq 0$.

(2) If $\limsup _{n \rightarrow \infty} y_{n}=T$, then there exists an integer $N \in \mathbb{N}^{+}$such that both the number sequences $\left\{y_{N+2 t}\right\}$ and $\left\{x_{N+2 t+k+1}\right\}$ are monotonically decreasing, and $y_{N+2 t} y_{N+2 t-1}=$ $1, x_{N+2 t+k} x_{N+2 t+k+1}=1$ for all $t \geq 0$.

Proof. Here, we only prove (1). By Lemma 5, if $\limsup \operatorname{sum}_{n \rightarrow \infty} x_{n}=T$ and $\lim \sup _{n \rightarrow \infty} y_{n}=M \leq T$, then there exists $N \in \mathbb{N}^{+}$such that $x_{N+2 t} \geq x_{N+2 t+2} \geq T$ for all $t \geq 0$. So $\left\{x_{N+2 t}\right\}$ is monotonically decreasing for all $t \geq 0$. There exists a constant $\varepsilon>0$ such that $a y_{N+2 t-k} \leq T-\varepsilon$. Hence,

$$
\begin{aligned}
\frac{1}{x_{N+2 t-1}} & \leq x_{N+2 t}=\max \left\{\frac{1}{x_{N+2 t-1}}, \frac{A_{N+2 t-1}}{y_{N+2 t-k-1}}\right\} \\
& \leq \max \left\{\frac{1}{x_{N+2 t-1}}, b y_{N+2 t-k}\right\} \\
& \leq \max \left\{\frac{1}{x_{N+2 t-1}}, T-\varepsilon\right\} \leq \frac{1}{x_{N+2 t-1}} .
\end{aligned}
$$

This implies $x_{N+2 t} x_{N+2 t-1}=1$. Without loss of generality, we assume $y_{N+2 t+k}<T / a$, so

$$
\begin{aligned}
\frac{1}{y_{N+2 t+k}} & \leq y_{N+2 t+k+1}=\max \left\{\frac{1}{y_{N+2 t+k}}, \frac{B_{N+2 t+k}}{x_{N+2 t}}\right\} \\
& =\frac{1}{y_{N+2 t+k}} \leq y_{N+2 t+k-1} .
\end{aligned}
$$

Hence, $y_{N+2 t+k} y_{N+2 t+k+1}=1$, and $\left\{y_{N+2 t+k+1}\right\}$ is monotonically decreasing for all $t \geq 0$. This completes the proof of the Lemma.

Theorem 7. Let $\left\{\left(x_{n}, y_{n}\right)\right\}_{n=-k}^{+\infty}$ be a solution of system (5). If $k$ is even, then one of the $\left\{x_{n}\right\}$ and $\left\{y_{n}\right\}$ is eventually periodic with period 2.

Proof. By Lemma 6, if $\lim \sup _{n \rightarrow \infty} x_{n}=T$, then there exists $N \in \mathbb{N}^{+}$such that both $\left\{x_{N+2 t}\right\}$ and $\left\{y_{N+2 t+k+1}\right\}$ are monotonically decreasing. We can easily get $x_{N+2 t} x_{N+2 t-1}=$ 1 and $y_{N+2 t+k} y_{N+2 t+k+1}=1$ for all $t \geq 0$.

We will prove that $\left\{y_{n}\right\}$ is eventually periodic with period 2. Otherwise, there will be infinitely many $k_{j}$ such that

$$
\begin{aligned}
y_{N+2 k_{j}+1} & =\max \left\{\frac{1}{y_{N+2 k_{j}}}, \frac{B_{N+2 k_{j}}}{x_{N+2 k_{j}-k}}\right\}=\frac{B_{N+2 k_{j}}}{x_{N+2 k_{j}-k}} \\
& >\frac{1}{y_{N+2 k_{j}}} .
\end{aligned}
$$

We take a subsequence $\left\{B_{N+2 k_{j}}\right\}$ and assume $B_{N+2 k_{j}} \rightarrow B \leq a$, $y_{N+2 k_{j}} \rightarrow L$. Since $-k$ is even, $x_{N+2 k_{j}-k} \rightarrow T$. Take the limit on both sides of the inequality $B_{N+2 k_{j}} / x_{N+2 k_{j}-k}>1 / y_{N+2 k_{j}}$; hence $B L>T$. Since $T \geq L, 1>a \geq B$, we have $a T \geq B L$, so
$T>a T \geq B L>T$. This is a contradiction. Therefore $\left\{y_{n}\right\}$ is eventually periodic with period 2 .

Similarly, we obtain that if $\lim _{\sup _{n \rightarrow \infty}} y_{n}=T$, then $\left\{x_{n}\right\}$ is eventually periodic with period 2 . This completes the proof of the theorem.

Theorem 8. If both $A_{n}$ and $B_{n}$ are periodic sequences, then the solution $\left\{\left(x_{n}, y_{n}\right)\right\}_{n=-k}^{+\infty}$ of system (5) converges to a period two solution.

Proof. We will only prove the case of $\limsup _{n \rightarrow \infty} x_{n}=T$. By Lemma 6, we know that if $\lim _{\sup _{n \rightarrow \infty}} x_{n}=T$, then there exists $N \in \mathbb{N}^{+}$such that both $\left\{x_{N+2 t}\right\}$ and $\left\{y_{N+2 t+k+1}\right\}$ are monotonically decreasing. We can get $x_{N+2 t} x_{N+2 t-1}=1$ and $y_{N+2 t+k} y_{N+2 t+k+1}=1$ for all $t \geq 0$ easily. So both $\left\{x_{N+2 t-1}\right\}$ and $\left\{y_{N+2 t+k}\right\}$ are monotonically increasing.

Now, we prove that $\left\{x_{n}\right\}$ is eventually periodic with period 2. Assume that $\left\{x_{n}\right\}$ is not eventually periodic with period 2 ; then $\left\{x_{N+2 t-1}\right\}$ is not an eventual constant, and there exist infinitely many $k_{j} \rightarrow+\infty$ such that

$$
\begin{aligned}
x_{N+2 k_{j}-1} & =\max \left\{\frac{1}{x_{N+2 k_{j}-2}}, \frac{A_{N+2 k_{j}-2}}{y_{N+2 k_{j}-k-2}}\right\} \\
& =\frac{A_{N+2 k_{j}-2}}{y_{N+2 k_{j}-k-2}}>\frac{1}{x_{N+2 k_{j}-2}} .
\end{aligned}
$$

Therefore

$$
A_{N+2 k_{j}-2}=x_{N+2 k_{j}-1} y_{N+2 k_{j}-k-2} \text {. }
$$

By Lemma 6, we know that $\left\{x_{N+2 k_{j}-1} y_{N+2 k_{j}-k-2}\right\}$ is monotonically increasing, not an eventual constant, so $\left\{A_{N+2 k_{j}-2}\right\}$ is monotonically increasing, not an eventual constant. This is a contradiction. So $\left\{x_{n}\right\}$ is eventually periodic with period 2 .

Similarly, we obtain that $\left\{y_{n}\right\}$ is eventually periodic with period 2. So $\left\{\left(x_{n}, y_{n}\right)\right\}_{n=-k}^{+\infty}$ converges to a period two solution. This completes the proof of the theorem.

\section{Conflicts of Interest}

The authors declare that they have no conflicts of interest.

\section{Authors' Contributions}

All the authors read and approved the final manuscript.

\section{References}

[1] W. J. Briden, E. A. Grove, and G. Ladas, "On the Nonautonomous Equation $x_{n+1}=\max \left\{A_{n} / x_{n}, B_{n} / x_{n-1}\right\}$," in Taiwan: Proceedings of the Third International Conference on Difference Equations, pp. 49-73, September 1997.

[2] E. A. Grove, C. M. Kent, and G. Ladas, "On $x_{n+1}=$ $\max \left\{1 / x_{n}, A_{n} / x_{n-k}\right\}$ with a period-3 parameter," Fields Institute Communications, vol. 29, pp. 133-139, 2001.

[3] V. L. Kocic and G. Ladas, Global Behavior of Nonlinear Difference Equations of Higher Order with Application, Kluwer Academic, Dordrecht, The Netherlands, 1993. 
[4] M. R. S. Kulenovic and G. Ladas, Dynamics of The Second Rational Difference Equations with Open Problems and Conjectures, Chapman and Hall, CRC, New York, NY, USA, 2002.

[5] G. Ladas, "Open problems and conjectures," Journal of Difference Equations and Applications, vol. 2, no. 3, pp. 339-341, 1996.

[6] D. g. Simsek, B. Demir, and C. Cinar, "On the solutions of the system of difference equations $x_{n+1}=\max \left\{A / x_{n}, y_{n} / x_{n}\right\}, y_{n+1}=$ $\max \left\{A / y_{n}, x_{n} / y_{n}\right\}, "$ Discrete Dynamics in Nature and Society, vol. 2009, Article ID 325296, 11 pages, 2009.

[7] S. Stevic, "On the recursive sequence $x_{n+1}=\max \left\{c, x_{n}^{p} / x_{n-1}^{p}\right\}$," Applied Mathematics Letters, vol. 21, no. 8, pp. 791-796, 2008.

[8] I. Szalkai, "On the periodicity of the sequence $x_{n}=$ $\max \left\{A_{0} / x_{n}, A_{1} / x_{n-1}, \ldots, A_{k} / x_{n-k}\right\}, n=0,1,2, \ldots$, , Journal of Difference Equations and Applications, vol. 5, no. 1, pp. 25-29, 1999.

[9] Y. Chen, "Eventual periodicity of $x_{n+1}=\max \left\{1 / x_{n}, A_{n} / x_{n-1}\right\}$ with periodic coefficients," Journal of Difference Equations and Applications, vol. 11, no. 15, pp. 1289-1294, 2005.

[10] X. Yang, X. Liao, and C. Li, "On a difference equation wtih maximum," Applied Mathematics and Computation, vol. 181, no. 1, pp. 1-5, 2006.

[11] T. Sun, B. Qin, H. Xi, and C. Han, "Global behavior of the maxtype difference equation $x_{n+1}=\max \left\{1 / x_{n}, A_{n} / x_{n}-1\right\}$," Abstract and Applied Analysis, vol. 2009, Article ID 152964, 10 pages, 2009. 


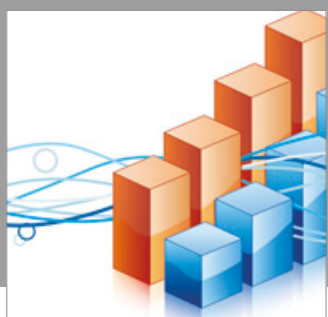

Advances in

Operations Research

vatersals

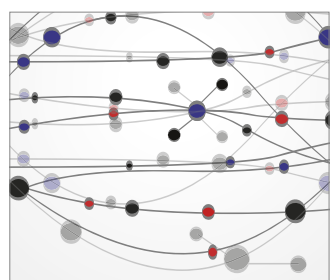

\section{The Scientific} World Journal
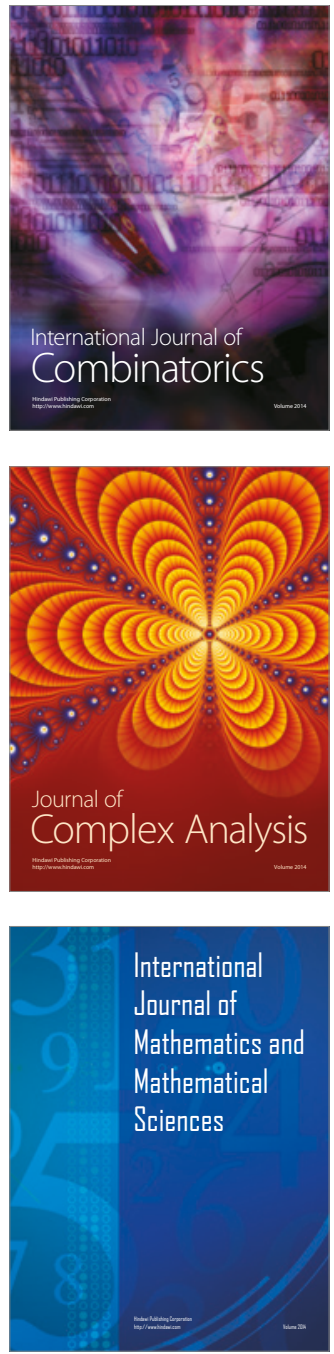
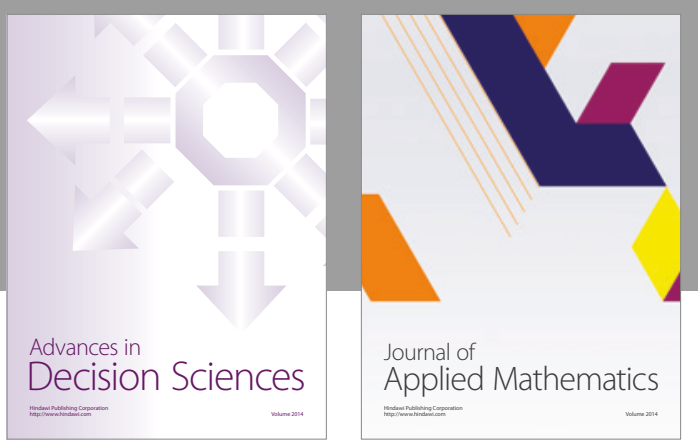

Algebra

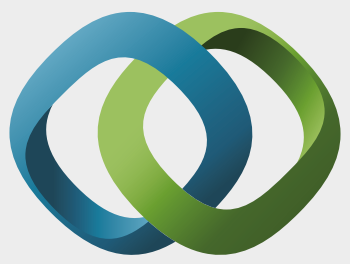

\section{Hindawi}

Submit your manuscripts at

https://www.hindawi.com
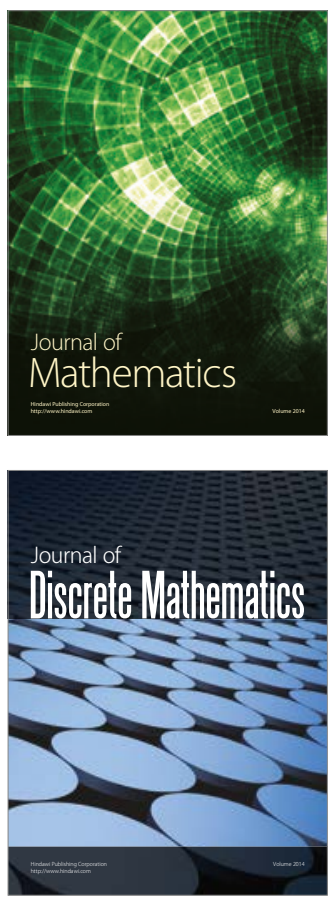

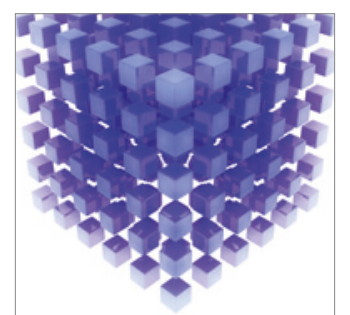

Mathematical Problems in Engineering
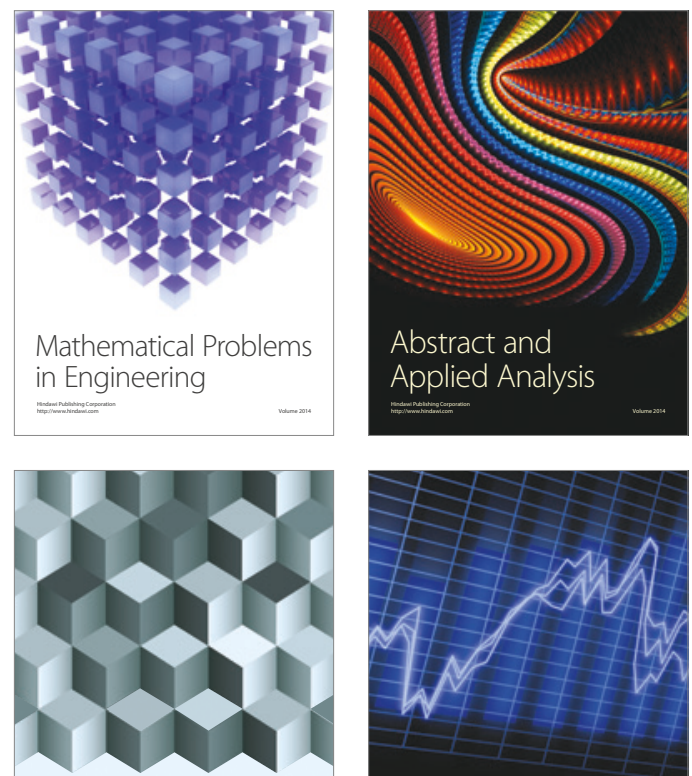

Journal of

Function Spaces

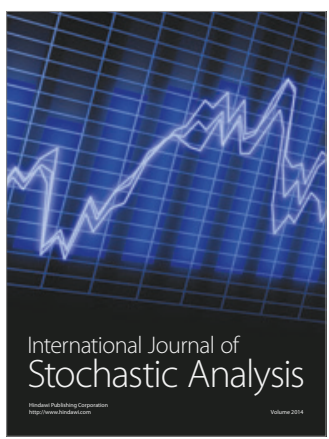

Probability and Statistics
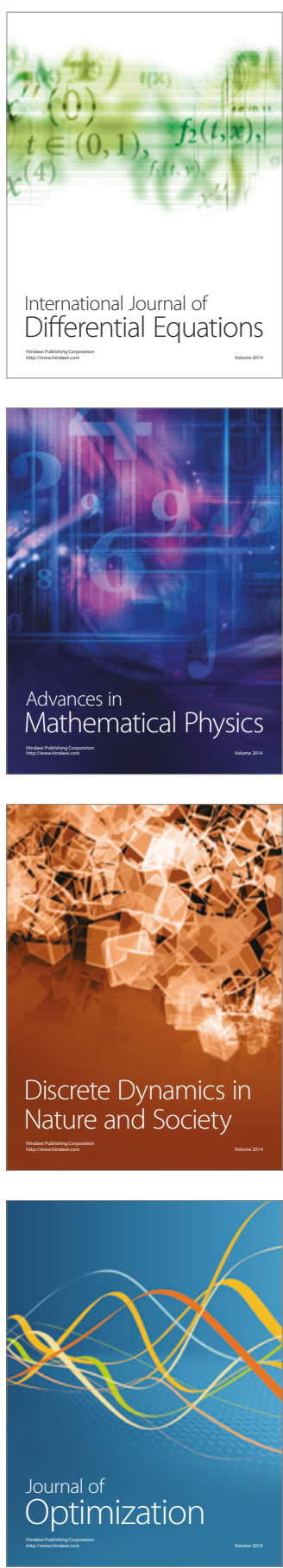